\title{
Kirstin Drenkhabn
}

\section{Die Lage der Kriminologie in Deutschland - ein Kommentar}

Ende Juni 2012 fand am Max-Planck-Institut für ausländisches und internationales Strafrecht in Freiburg eine Tagung „zur Lage der Kriminologie in Deutschland“ statt, deren greifbarstes Ergebnis die in diesem Heft abgedruckte Freiburger Erklärung ist. Ich war auf dieser Tagung eine der wenigen Personen ohne Lehrstuhl, ansonsten waren aus der nachwachsenden Generation einige Doktorandinnen und Doktoranden und auch einige Mitarbeiterinnen und Mitarbeiter des MPI dabei. Mit diesem Kommentar stelle ich meine Bewertung der Lage als Nachwuchskriminologin vor. Ich kann dabei nicht für „den Nachwuchs" sprechen, denn es gibt keine gemeinsame Linie. Allerdings weiß ich, dass viele aus dieser Altersgruppe vieles ähnlich sehen.

Auf der Tagung sollte also die aktuelle Lage besprochen werden, es gab eine Vielzahl von Vorträgen, die m. E. zum Teil weit über das hinausgingen, was für eine Lagebeschreibung und eine Strategiediskussion erforderlich gewesen wäre (Programm: http:// www.mpicc.de/shared/data/pdf/programm_kriminologie_de_12.pdf). Zumindest der Vortragsblock über neue Forschungsfelder hätte - auch wenn die Beiträge interessant waren - nicht sein müssen. Die Lage der Kriminologie in Deutschland ist nicht durch einen Mangel an Themen, sondern durch einen eklatanten Mangel an Forscherinnen und Forschern gekennzeichnet.

Das ist bei einer Bevölkerung in der Bundesrepublik Deutschland von über 80 Millionen und einer weiten Hochschullandschaft ein merkwürdiger Befund. Man sollte meinen, dass unter diesen Voraussetzungen auch ausreichend wissenschaftliches Personal für die Erforschung von Phänomenen hervorgebracht werden sollte, die die Öffentlichkeit so sehr umtreiben, wie es Kriminalität und abweichendes Verhalten tun. Dieses Rätsel wurde auf der Tagung in Ansätzen gelöst: In den Bezugswissenschaften sterben mit dem Ausscheiden der Lehrstuhlinhaber aus dem aktiven Dienst die Lehrstühle mit einer entsprechenden Denomination ab. Selbst wenn diese Stellen nicht vollständig wegfallen, werden Kriminologie, abweichendes Verhalten, Rechtspsychologie und ähnliche Stichwörter in Neuausschreibungen nicht genannt oder nicht besonders herausgestellt. Es ist dann dem Zufall bzw. der Berufungskommission überlassen, jemanden auszuwählen, der/die irgendwie auf die Ausschreibung passt und sich heimlich für kriminologische Themen interessiert. Wie viele heimliche KriminologInnen es gibt, lässt sich nicht sagen, da viele sich selbst gar nicht so definieren werden und damit in einer anderen wissenschaftlichen community beheimatet sind. In der Psychologie, Soziologie und Psychiatrie hat diese Entwicklung schon zu einer weitgehenden Verdrängung bekennender KriminologInnen geführt. Auch an den Fachhochschulen gibt es viele KollegIn- 
nen, die sich mit kriminologischen Themen beschäftigen, allerdings fehlen hier vielen die Zeit und das Personal für umfangreiche Forschung.

Als Kriminologin mit juristischer Ausbildung interessiert mich aber vor allem, was an den rechtswissenschaftlichen Fachbereichen passiert. Hier kann man schon von einer Art Backlash sprechen: Während ab den 1970er Jahren viele Fachbereiche im Strafrecht kriminologische Lehrstühle einrichteten, werden diese Stellen heute häufig eingespart oder dem materiellen Strafrecht gewidmet. Zwar taucht Kriminologie als eines unter mehreren auch mit zu vertretenden Nebenfächern noch in Ausschreibungen auf, allerdings heißt das im deutschen Strafrecht nicht viel. Hier macht sich nämlich noch ein weiterer Fallstrick bemerkbar: Den NachwuchsforscherInnen, die es wagen, in der ersten Qualifikationsarbeit irgendetwas erkennbar kriminologisches zu untersuchen, wird nachdrücklich geraten, die zweite Arbeit einem dogmatischen Thema zu widmen - sonst wird es mit der Strafrechts-Venia schwierig, ohne die man kein ordentlicher Mensch ist. Bei Menschen, die es „richtig“ gemacht haben und eine Arbeit zum materiellen Strafrecht und eine zum Prozessrecht geschrieben haben, scheint hingegen die Venia für Kriminologie günstig zu haben zu sein - man wundert sich schon manchmal, wer dieses Fach zukünftig alles wird lehren dürfen.

Dahinter verbirgt sich natürlich auf Seiten der dogmatischen StrafrechtlerInnen auch die verständliche Befürchtung, die Pflichtfachlehre nicht mehr abdecken zu können, wenn einer oder eine von drei oder vier aus der Reihe tanzt. Ich meine aber, dass dahinter noch mehr steckt. Einige bezeichnen Kriminologie (immer noch?) als Erbsen- oder Fliegenbeinzählerei. Das zeigt natürlich vor allem, dass sie keine Ahnung haben. Dies zu wissen, hilft aber als Bewerberin in einem Berufungsverfahren nicht weiter, denn in der Kommission wird kein Erbsen- bzw. Fliegenbeinzähler sitzen - gilt es für diese Person doch gerade eine Nachfolge zu finden. Vielleicht zeigt diese Herabwürdigung auch, dass Kriminologie bei den anderen im gedanklichen Mitbewusstsein gar nicht vorhanden ist, weil dieses Fach z. B. auf der Strafrechtslehrertagung nicht wahrnehmbar ist. Es zeigt aber auch, dass es anscheinend uns juristischen KriminologInnen nicht gelungen ist, die Bedeutung unserer Forschung für die Strafrechtswissenschaft herauszustellen.

Woran liegt das? Die Antwort führt wieder zum Venia- und Habilitationsproblem zurück. Viele StrafrechtlerInnen sehen Kriminologie allenfalls als Hilfswissenschaft an, die ein paar Zahlen zuliefert. KriminologInnen sehen die Kriminologie als eigene Wissenschaft mit eigenen Themen und eigenen Methoden an. Unsere Methoden unterscheiden sich grundlegend von denen des dogmatischen Strafrechts - es handelt sich vor allem um solche der empirischen Sozialforschung, für die Wissen erforderlich ist, das man im juristischen Studium und in der dogmatischen Arbeit nicht erwirbt. Das gleiche gilt für die Planung empirischer Forschungsvorhaben - auch das dafür erforderliche Wissen muss außerhalb des juristischen Studiums erworben werden. Für juristische KriminologInnen in Deutschland bedeutet dies, dass sie sich in der Qualifikationsphase völlig neue Kenntnisse aneignen und sich spezialisieren müssen, wenn sie gute kriminologische Forschung leisten wollen. Außerdem müssen sie den Austausch mit KollegInnen aus dem Ausland pflegen und in englischsprachigen Zeitschriften veröffentlichen, wenn sie den Anschluss an die Forschung nicht verpassen wollen - der Takt wird in unserer Wissen-

NK 25. Jg. 1/2013 
schaft nicht in Deutschland vorgegeben. Damit macht man sich aber zu Hause nicht eben berufungsfähig: Zeit für ein Buch über ein dogmatisches Thema hat man nicht, potenzielle Mitglieder einer Berufungskommission werden Schwierigkeiten haben, empirische Forschung nachzuvollziehen (Zahlen! Berechnungen!), und Englisches wird scheinbar nicht gelesen. Hinzu kommt, dass das Habilitationsritual („Schreiben Sie doch einfach endlich dieses Buch! Liest doch sowieso keiner.") von den KriminologInnen, zumal aus dem Ausland, nicht verstanden wird. Andernorts geht man davon aus, dass Monographien geschrieben werden, wenn es wirklich etwas zu sagen gibt. Außerdem leben Verlage dort vom Verkauf der Bücher und nicht vom Druck. Man sitzt also als Nachwuchskriminologin zwischen Baum und Borke und behält am besten den Arbeitsmarkt in Belgien, den Niederlanden und dem Vereinigten Königreich fest im Blick.

Aber soll das wirklich die Lösung sein: aufgeben oder auswandern? Mal davon abgesehen, dass wir keine „Kriminalpolitik im Blindflug“ wollen, liegen kriminologische Forschungsthemen förmlich auf der Straße, was man von dogmatischen Themen, vor allem im Kernstrafrecht, nicht sagen kann. Außerdem stellt die Kriminologie auch gerne mal kritische Fragen wie z. B. nach der Legitimität, dem Rückhalt von Strafrecht in der Gesellschaft (was die KriminologInnen bewegt, lässt sich z. B. an den Programmen der ESC-Konferenzen ablesen, http://www.esc-eurocrim.org). Anstatt die Kriminologie zu vertreiben oder auf das Vorlesen von Lehrbüchern zu beschränken (wer soll die eigentlich schreiben?), sollte sich die Strafrechtsdogmatik das Potenzial der Kriminologie zu Nutze machen, um neue Fragen aufzuwerfen und ihren eigenen Status als Wissenschaft abzusichern. Nur nebenbei sei hier auch das aktuelle Lieblingswort der Universitätsleitungen genannt: Drittmittel. Ein solches Zusammenspiel im Rahmen einer gesamten Strafrechtswissenschaft funktioniert aber nur, wenn man uns den Verzicht auf die dogmatische Arbeit zugesteht und uns schon in der Qualifikationsphase ganz in unsere Wissenschaft eintauchen lässt. 